\title{
Virtual Queueing, Techniques for UBR+ Service in ATM with Fair Accesis and Minimum Bandwidth Guarantee
}

\author{
Kai-Yeung Siu Yuan Wu Wenge Ren \\ Masisachusetts Institute of Technology
}

$$
\text { DOE/ER/25273--T1-Pt. } 2
$$

\begin{abstract}
-
The ATM Forum is currently discussing the need for a new best-effort service called UBR+, which is an enhancement to the existing UBR service, to support data traffic. The objective of the UBR+ service is to provide each user with a minimum service rate guarantee and a fair access to any excess available bandwidth. In this paper, we present a new efficient scheme for supporting this service. The key advantage of our scheme is that it employs only FIFO queueing (instead of per-VC queueing) and admits simple: implementation in ATM switches. Our ideas involve a simple scheduling mechanism that is based on per-VC queueing and incorporate the virtual queueing technique [10], [11] that can efficiently emulate per-VC queuing on a shared FIFO queue. Simulation results are presented to show that our schemes can deliver almost ideal performance for supporting the new service requirements of UBR+.
\end{abstract}

\section{INTRODUCTION}

While ATM was originally conceived as a carrier of integrated traffic, the recent momentum on the rajpid standardization of the technology has come from data networking applications. Since most data applications cennot predict their own bandwidth requirements, they usually require a service that allows all competing active virtual connections (VCs) to dynamically share the available bandwidth. The unspecified bit rate (UBR) and the available bit rate (ABR) service classes in ATM have been developed specifically to support data applications.

The UBR service is designed for those data applications that want to use any available bandwidth and are not sensitive to cell loss or delay. Such connections are not rejected on the basis of bandwidth shortage and not policed for their usage behavior. During congestion, the cells may be lost but the sources are not expected to reduce their cell rates. Instead, these applications may have their own higher-level loss recovery and retransmission mechanisms, such as the window flow control employed by TCP. The advantage of using UBR service for data transport is its simplicity and minimal interaction required between users and the ATM network.

The ABR service has been developed with the goal of minimizing switch buffer requirement and cell loss in transporting data and allowing users to have fair access to the available bandwidth. To achieve such service requirements, the ABR service uses congestion control at the ATM layer. It requires network switches to constantly moritor the traffic load and feed the information back to the sources. The

Please send all correspondences to K.Y. Siu at Rm. 3-250, 77 Massachusetts Ave., MIT, Cambridge, MA 02139. Ph. \& ]Fax: (617) 2535589. Email:siu@list.mit.edu. This work was supported in part by the NSF Young Investigator Awards Program (Grant \# MIP-9357553) and by the Department of Energy (Grant \#DE-FG03-95EIR25273.) Inc. sources are expected to adjust their input to the network dynamically based on the congestion status of the network.

In fact, there has been a continuing debate in the networking community about the need for ABR service. A major argument against $\mathrm{ABR}$ is that while $\mathrm{ABR}$ assumes that the end systems comply with the ABR source behavior, most current applications are only connected to ATM via legacy networks such as Ethernet. Therefore, ABR may only push congestion to the network edges and cannot provide flow control on an end-to-end basis. Simulation results reported in recent ATM Forum contributions [12], [3] seem to support this argument.

Furthermore, it has been argued in [5] that most users today are typically either not able to specify the range of traffic parameters needed to request most ATM services, or are not equipped to comply with the (source) behavior rules required by existing ATM services. As a result, there are many existing users for whom the benefits of ATM service guarantees remain unattainable. Those users access ATM networks mostly through UBR connections, which provide no service guarantees. In view of this, [5] proposes a new service called UBR+, which will provide users with some level of service guarantees yet require minimal interaction between users and ATM networks.

The UBR+ service specifies that a user should be provided with a minimum service rate guarantee ${ }^{1}$ and with fair access to any excess available bandwidth. In other words, the UBR+ service will guarantee a user with a minimum throughput when the network is congested, while allowing a user to send at a higher rate when additional bandwidth is available.

Our main contribution in this paper is to present a new efficient scheme for supporting the UBR+ service. The key advantage of our scheme is that it employs only FIFO queueing (instead of per-VC queueing) and admits simple implementation in ATM switches. Our scheme is based on a simple weighted round robin scheduling mechanism using perVC queueing that can support UBR+. We then apply a variation of the virtual queueing technique [10], [11] to emulate this scheduling mechanism on a shared FIFO queue.

\section{Related Works}

As we discussed earlier, there has been an intensive debate in the ATM Forum on the need for an enhancement to the existing UBR service that provides each user with a minimum service rate guarantee. The essential idea in [5] is that by monitoring the rate of each connection at the ingress of an ATM network and tagging an appropriate proportion of

\footnotetext{
${ }^{1}$ To be more precise, the minimum service rate is guaranteed under the assumption of a given maximum packet size (SDU).
} 


\section{DISCLAIMER}

This report was prepared as an account of work sponsored by an agency of the United States Government. Neither the United States Government nor any agency thereof, nor any of their emiployees, makes any warranty, express or implied, or assumes any legal liability or responsibility for the accuracy, completeness, or usefulness of any information, apparatus, product, or process disclosed, or represents that its use would not infringe privately owned rights. Reference herein to any specific commercial product, prixess, or service by trade name, trademark, manufacturer, or otherwise does not necessarily constitute or imply its endorsement, recommendation, or favoring by the United States Government or any agency thereof. The views and opinions of authors expressed herein do not necessarily state or reflect those of the United States Government or any agency thereof. 


\section{DISCLAIMER}

Portions of this document may be illegible in electronic innage products. Images are produced from the best available original document. 
the cells when the rate exceeds the negotiated rate, one can provide each connection with a minimum bandwidth guarantee. However, this scheme when implemented in switches with only FIFO queues cannot provide each coinnection with a fair share of the excess available bandwidth. It is further suggested in [5] that per-VC queueing may be necessary for supporting UBR+ service.

It is proposed in [6] that the service requirements of UBR+ can actually be achieved with a new conformance definition within the VBR service category, and therefore no new techniques need to be invented. However, as argued in [5], the advantage of the proposed UBR+ service is that it requires little or no involvement on the user part since most service parameters can be negotiated ofl-line, e.g., by network management, configuration means, or inferred from existing parameters in the setup phase.

This paper describes an efficient solution for supporting UBR+ service. Unlike the proposal in [5], our techniques can be implemented in switches with FIFO queues to provide users with fair access to the excess availatle bandwidth in addition to the minimum service rate guarantee. Moreover, our techniques eliminate the need for monitoring the rate of each connection at the ingress of an ATM network. Our results are based on the idea of virtual queueing as reported in several recent works [1], [10], [11].

The virtual queueing technique was first proposed in [1] to improve rate allocations for ABR connections in the presence of VBR traffic. This technique is applied in [10] to emulate the round robin service of per-VC queuing on a FIFO queue for improving the performance of TCl? over ATM. Simulation results in [10] illustrate that when combined with the EPD techniques [9], virtual queuing can provide significant improvement of fairness among TCP connections.

The virtual queuing technique utilizes the queue length on a per-VC basis in a shared FIFO queue to achieve fair buffer allocation. While this choice of per-VC state variable is quite intuitive and yields reasonably good performance, no analysis is given in [10] to provide a theoretical understanding of the technique. In [11], we propose a new choice of per-VC state variable, which has a precise analytical relation to the number of cells transmitted for each VC. The theoretical insights allow us to design an improved scheme that achieves nearly perfect fairness of average throughput among multiple TCP connections. In this paper, we shall apply a variation of the virtual queueing technique developed in [11] that can emulate a weighted round robin service on a FIFO queue.

\section{SCHEDULING BASED ON PER-VC QueueING}

We now describe a mechanism for scheduling UBR+ connections based on per-VC queueing.

In ATM networks, data traffic supported by UBR+ is usually mixed with other higher priority CBR/VBR traffic. Consequently, it is expected that the available bandwidth $B$ allocated to UBR+ connections will vary dynarnically. Since UBR + guarantees each connection a minimum cell rate (MCR), we shall assume that before admitting a connection, the network will use some admission control and bandwidth

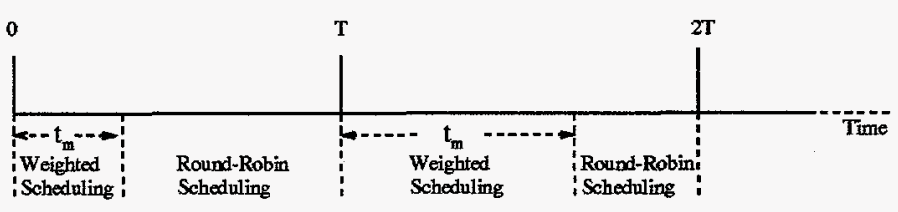

Fig. 1. Two phases of the scheduling mechanism

reservation procedure to ensure that the available bandwidth $B$ (in cells/sec) is always greater than or equal to the sum of the MCR of every VC, i.e., $B \geq \sum_{i=1}^{N} M C R_{i}$, where $M C R_{i}$ (in cells/sec) is the minimum cell rate of $V C_{i}$, and $\mathrm{N}$ is the number of UBR+ connections.

We first pick a fixed but arbitrary time period $T$ (in sec) such that $T \cdot M C R_{i} \geq 1$ for each $V C_{i}$. Our scheduling mechanism consists of two phases in each period $T$. In the first phase, the scheduler will serve $T \cdot M C R_{i}$ cells for each $V C_{i}$. In other words, the number of cells to be served during the first phase of each period $T$ is $T \cdot \sum_{i=1}^{N} M C R_{i}$. Note that since the available bandwidth is $B \geq \sum_{i=1}^{N} M C R_{i}$, the total number of cells that can be served during the period $T$ is $T$. $B \geq T \cdot \sum_{i=1}^{N} M C R_{i}$. Thus, after the first phase is complete, there are $E=T \cdot\left(B-\sum_{i=1}^{N} M C R_{i}\right)$ cells to be served within each period $\mathrm{T}$. These $E$ cells correspond to those that can be served whenever there is left-over bandwidth after serving each VC at its minimum guaranteed cell rate. In the second phase of each period $T$, the scheduler simply serves each $\mathrm{VC}$ in a round robin fashion to achieve fair allocation of the left-over bandwidth.

Figure 1 illustrates the two phases of the scheduling mechanism. The time axis is divided into consecutive time periods of length $T$. In the figure, $t_{m}$ denotes the time it takes to serve the minimum guaranteed traffic of all VCs within each period $T$. Since the available bandwidth $B$ for the UBR+ connections is constantly varying, the time $t_{m}$ also varies between two different periods. But we always have $t_{m} \leq T$ because $\sum_{i=1}^{N} M C R_{i} \leq B$. During the remaining time $\left(T-t_{m}\right)$ within each period $T$, the excess bandwidth can be shared equally among the VCs simply by serving the VCs in a round robin fashion.

There are several ways of implementing the MCR scheduling phase. Our idea is to associate each $V C_{j}$ with a weight corresponding to the number of cells $\left(T \cdot M C R_{j}\right)$ that should be served within each period $T$ to guarantee its MCR. We next present the pseudocode for the MCR scheduling algorithm based on per-VC queueing.

MCR scheduling based on per-VC queueing We assume a separate queue is maintained for each active VC. A register $W_{i}$ with initial value 0 is maintained for each $V C_{j}$, whose $M C R_{i}$ (in cells/sec) is given. A time period $\mathrm{T}$ (in sec) is chosen such that $T \cdot M C R_{i} \geq 1$ for each $i$. (Note $T \cdot M C R_{i}$ needs not be an integer.)

$$
\begin{aligned}
& \text { For } j=1,2, \ldots, \mathrm{N} \\
& \quad W_{j}=W_{j}+M C R_{j} \times T \\
& \text { While } W_{i} \geq 1 \text { for at least some } V C_{i} \\
& \quad \text { If } W_{j} \geq 1 \text { AND the queue of } V C_{j} \text { is non-empty }
\end{aligned}
$$




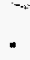

serve one cell of $V C_{j}$

$W_{j}=W_{j}-1$

go to the next VC

End of MCR Scheduling

The above MCR scheduling algorithm requires that any VC can be served at any time, and thus relies heavily on the use of per-VC queueing. In subsequent sections, we shall show how to approximate the above scheduling algorithm with the use of a shared FIFO queue. To help understand our scheme, we next discuss the ideas of early packet discard (EPD) mechanism and its role in our scheduling mechanism.

\section{EPD and Fair Buffer Allocation 'Techniques}

As we discussed earlier in the introduction, UBR service does not specify any congestion control mechanism at the ATM layer. When TCP or UDP traffic is transported over UBR, cells are simply discarded when there is a buffer shortage during network congestion. Since each disicarded cell is likely to belong to a different packet, a significant number of transmitted packets may have already been corrupted by cell loss and thus need to be retransmitted, resulting in a substantial waste of bandwidth and degradation of throughput.

To address this problem, more sophisticated frame discarding mechanisms such as the early packet liscard (EPD) algorithm [9] for UBR service have been proposed. The basic idea of EPD is to discard an entire packet prior to buffer overflow, so that the bandwidth is only utilized for the transmission of noncorrupted packets.

A simple way to implement the EPD mechanism in a shared FIFO queue is to set a threshold value (EPD threshold). An incoming packet will be dropped whenever the queue length exceeds the preset threshold value. To be more specific, the first cell of an incoming packet will be discarded if the total queue length at an ATM switch exceeds the EPD threshold. Once the first cell of a packet is discarded, the remaining cells of this packet will also be discarded, even when the queue length reduces below the EJPD threshold. However, a cell will not be discarded by the algorithm if the first cell of the same packet has already been accepted into the queue, so that packet integrity is preserved. Thus, with EPD, the switch delivers either all or none of the cells of an incoming packet, to ensure that no bandwidth is wasted in delivering corrupted partial packets. Hence, UBR with EPD achieves higher bandwidth utilization than plain UBR service. The EPD threshold should be chosen so that the excess buffer (difference between total buffer size and EPD threshold) is large enough to ensure a low rate of buffer overflow.

A drawback of the EPD scheme described above is that it cannot achieve fair bandwidth allocation among competing VCs [7]. For example, EPD tends to allocate less bandwidth for VCs traversing multiple congested nodes.

One method for improving the fairness of packet-based traffic over UBR with EPD is to use per-VC queueing [8]. The idea is that by controlling the buffer allocation for each VC, EPD can serve as a mechanism to control the through- put of each VC. In particular, when the total queue occupancy exceeds the EPD threshold, an incoming packet will be discarded if it belongs to a VC whose queue length is greater than or equal to its fair share of the buffer (e.g., the average queue length). In other words, the first cell of a packet belonging $V C_{j}$ with queue length $Q_{j}$ is discarded if: 1) the current total queue length $Q \geq T h$, and 2) $Q_{j} \geq \overline{T h}$ where $\overline{T h}$ can be set to a predetermined value such as $T h / N$ or a dynamic parameter such as $Q / N$ ( $\mathrm{N}$ is the number of active VCs that have cells in the buffer). It is easy to see that per-VC queueing improves fairness since cells are only discarded from connections that have used more resources than their fair share. This is clearly better than discarding the cells randomly as with simple EPD. Moreover, by buffering the cells of each VC on a separate queue, one can schedule cell transmission for each $\mathrm{VC}$ at the output in a round-robin fashion.

\section{Scheduling based on Vtrtual Queueing}

Virtual queuing emulates the round-robin buffer allocation provided by per-VC queuing on a shared FIFO queue. More specifically, a separate "virtual" queue is implemented for each VC by maintaining a state variable $M_{j}$ for each $V C_{j}$. The variable $M_{j}$ is increased by one whenever a cell of $V C_{j}$ is admitted to the FIFO queue. When the cells are transmitted out of the FIFO queue, however, the variables $M_{j}$ 's are decremented in a round-robin fashion, regardless of which VC the cell transmitted actually belongs to. Thus, $M_{j}$ does not correspond to the actual queue occupancy of $V C_{j}$ in the FIFO queue. Instead, it represents the queue occupancy of $V C_{j}$ as if per-VC queuing and round-robin output scheduling are implemented. The EPD mechanism is then applied to the incoming packets as in the case with per-VC queueing, except that the virtual queue $M_{j}$ is used instead of the actual queue length $Q_{j}$ for each VC.

The following pseudocode describes the cell discarding policy implemented at the input port of a switch based on virtual queueing:

\section{Discarding policy based on virtual queueing} We associate each $V C_{j}$ with a virtual queue variable $M_{j}$. $L$ is a list containing the identifiers of the active VCs. The variable $Q$ denotes the total queue length of the FIFO queue.

When a cell in $V C_{i}$ reaches a switch:

if the cell is the first cell of a packet

$\overline{T h}:=T h / N$

if $Q \geq T h$ and $M_{i} \geq \overline{T h}$

discard this cell

else

accept this cell into the FIFO queue

$M_{i}:=M_{i}+1$

if $M_{i}=-w+1$

append $i$ to the tail of $L$

else

if any cell of the packet has been discarded discard this cell

else

if $Q \geq Q_{\max }$ 
discard this cell

else

accept this cell into the FIFO queue

$M_{i}:=M_{i}+1$

if $M_{i}=-w+1$

append $i$ to the tail of $L$

In the above algorithm, a lower bound - $v$ (a negative value) needs to be imposed on $M_{j}$ because pickets of $V C_{j}$ are accepted into the buffer as long as $M_{j}$ is less than the discard threshold $(\overline{T h})$. If $M_{j}$ is unbounded and becomes very negative, a large burst of incoming packets of $V C_{j}$ will be accepted, which could result in buffer overflow.

Recall from Section III that our scheduling mechanism consists of 2 phases within each consecutive period $T$. The first phase deals with MCR scheduling and the other with round robin scheduling for sharing the excess bandwidth. We now apply the virtual queueing technique to emulate the per-VC MCR scheduling presented in Section III. The following pseudocode describes the algorithm. We use the same notations as introduced earlier.

For $j=1,2, \ldots, \mathrm{N}$

$W_{j}=W_{j}+M C R_{j} \cdot T$

When a cell of the FIFO queue is transmitted

if $L$ is not empty

let $i$ denote the first connection identifier in $L$ remove $i$ from $L$

$M_{i}=M_{i}-1$

$W_{i}=W_{i}-1$

if $M_{i}>-w$ AND $W_{i}>1$

append $i$ to the tail of $L$

If $W_{i}<1$ for all VCs

End of MCR Scheduling

It remains to be shown how the excess bandwidth can be shared equally among the VCs. The pseudocode below describes the round-robin service emulated by virtual queueing:

When a cell of the FIFO queue is transmitted

if $L$ is not empty

let $i$ denote the first connection identifier in $L$ remove $i$ from $L$

$M_{i}:=M_{i}-1$

if $M_{i}>-w$

append $i$ to the tail of $L$

We have completed the description of our scheme for supporting UBR+ service with FIFO queueing. In the remainder of this paper, we shall present a simulation study of the performance of our scheme.

\section{Simulation Model and Parameters}

Our simulation tool is based on the MIT Network Simulator (NetSim). NetSim is an event-driven simulator composed of various components that send messages to one an-

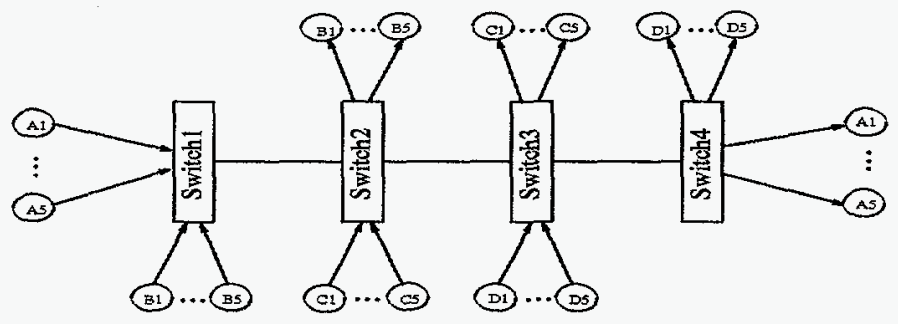

Fig. 2. A chain configuration.

other. We have built upon the NetSim package various ATM and TCP/UDP related components. These components include an ATM switch component, a SONET OC-x link component, an ATM host component, a greedy/bursty source component, a multiplexing and demultiplexing component, a UDP component, and a TCP component. In our simulations, we have a fine TCP timer granularity, which is $10 \mu \mathrm{sec}$, instead of 300 to $500 \mathrm{msec}$ as in most TCP releases. The ATM host component performs ATM Adaptation Layer for data services (AAL5) including segmentation and reassembly (SAR) of TCP packets. The ATM switch component models a UBR switch combined with early packet discarding capability. Each output port has a single FIFO queue, i.e., an output buffer.

Figure 2 illustrates the simulation model of a network with 20 persistent data connections in a chain configuration. For simplicity, instead of illustrating the details of the components, we group user, TCP/UDP, and ATM host components into a single node in this figure. Note that VC A1-A5 traverse three congested links but each of the other VCs (B1-B5, C1-C5, D1-D5) traverses only one congested link. Moreover, each link is traversed by $10 \mathrm{VCs}$ and is assumed to have the same capacity (150Mbps).

In our simulations, we consider a link delay of $500 \mathrm{nsec}$ (about the distance of $100 \mathrm{~m}$ ) to model a LAN environment. We expect TCP performance to be similar as long as the link distance lies within $L A N$ range, e.g., up to $1000 \mathrm{~m}$. This is because even with a $1000 \mathrm{~m}$ link distance, the link delay is only $5 \mu \mathrm{sec}$, which is negligible compared with the TCP packet processing delay of $300 \mu \mathrm{sec}$ in our simulation.

\section{Simulation Results}

We now present simulation results with the chain configuration. Note that connections A1-A5 traverse multiple links while each of the other connections (B1-B5, C1-C5, D1-D5) traverses only a single link.

Simulation results in Figure 3 show that our scheme provides almost perfect fairness and throughput when UDP sources are used. Each VC's MCR is guaranteed and the excess bandwidth is distributed fairly. These simulation results show that with greedy UDP sources, each user can get its fair rate very quickly. Although the simulation is done over a 2 second period, we note that the throughput of each connection is quite uniform over any period of time, In particular, the results regarding the throughput and the fairness will remain essentially the same if we only perform the simulation over the first 50 msec period. 

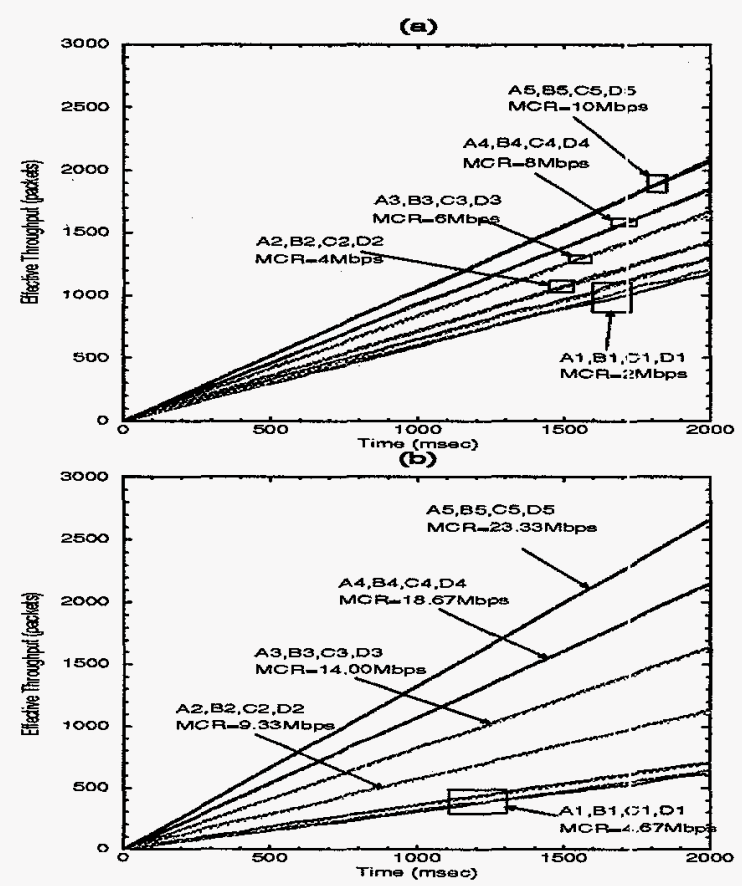

Fig. 3. UDP traffic in the Chain Configuration.

The preceding simulation results using UDP traffic clearly illustrate the fairness properties of our scherre at the cell level. Since many data applications today employ TCP as their transport layer protocol, we have also performed simulations with TCP traffic using the chain configuration. Because of the window flow control mechanism used in TCP, the packet-level throughput and fairness performance of our scheme will be different from UDP traffic. Jior example, UDP sources can constantly transmit traffic at the peak cell rate (PCR) whereas TCP sources have to go through a slow-start phase when packets are lost. But our simulation results employing TCP sources in the chain configuration still show very good fairness and throughput, as shown in Figure 4.

\section{Concluding Remarks}

We have presented a new efficient scheme for supporting the service requirements of UBR+. Unlike other scheme [5] proposed in the ATM Forum, our technique does not require the use of per-VC queueing for fair access to the excess bandwidth. It incorporates the EPD mechanism and a fair buffer allocation technique called virtual queueing to guarantee a user with a minimum bandwidth. Moreover, our scheme admits simple implementation in ATM switches. We have also presented simulation results to show that our scheme can deliver almost ideal performance in terms of the UBR+ service requirements.

\section{REFERENCES}

[1] F. Chiussi, Y. Xia, and V. P. Kumar. Virtual Queuing Techniques for ABR Service: Improving ABR/VBR Interaction. IEEE Infocom'97.

[2] V. Jacobson. Congestion Avoidance and Control. Proc. ACM SIGCOMM'88, pages 314-329, August 1988.
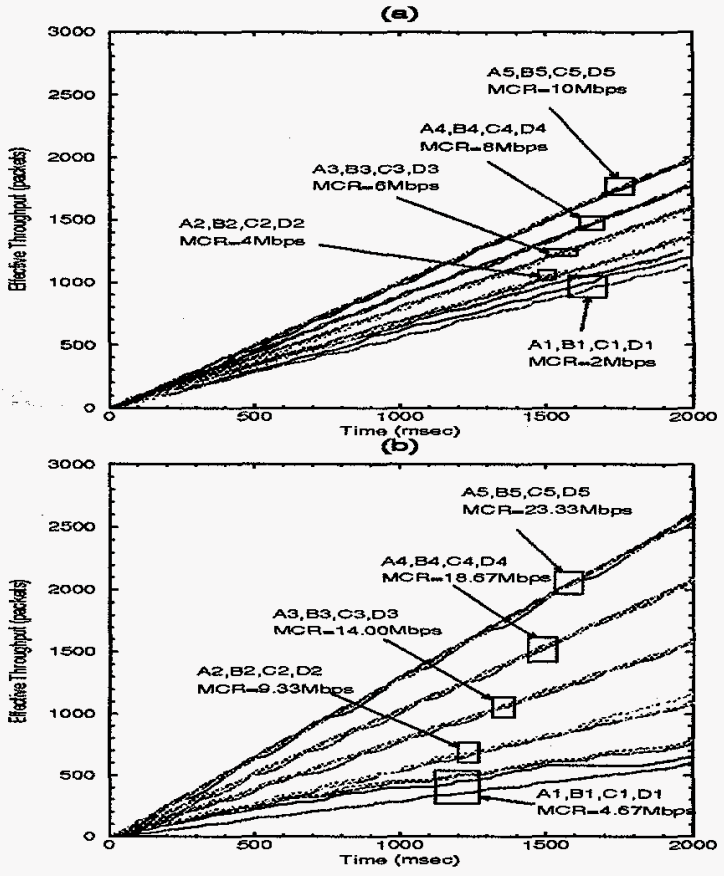

Fig. 4. TCP traffic in the Chain Configuration.

[3] S. Jagannath and N. Yin. End-to-End TCP Performance in IP/ATM Internetworks. ATM Forum Contribution 96-1711, December 1996.

[4] R. Jain The Art of Computer Systems Performance Analysis: Techniques for Experimental Design, Measurement, Simulation, and Modeling, Wiley- Interscience, New York, NY, April 1991.

[5] R. Guerin and J. Heinanen. UBR+ Service Category Definition. ATM Forum Contribution 96-1598, December 1996.

[6] J. B. Kenney Satisfying UBR+ Requirements via a New VBR Conformance Definition. ATM Forum Contribution 97-0185, February 1997.

[7] H. Li, K.-Y. Siu, H.-Y. Tzeng, C. Tkeda and H. Suzuki. A Simulation Study of TCP Performance in ATM Networks with ABR and UBR Services. Proceedings of IEEE INFOCOM'96, March 1996. A journal version of this paper is to appear in the IEEE Transactions on Communications, Special Issue on High Speed Local Area Network, May 1996.

[8] H. Li, K.-Y. Siu, H.-Y. Tzeng, C. Ikeda and H. Suzuki. Performance of TCP over UBR Service in ATM Networks with Per-VO Early Packet Discard Schemes. Proceedings of the International Phoenix Conference on Computers and Communications, March 1996. A journal version of this paper is to appear in Computer Communications, Special Issue on Enabling ATM Networks.

[9] A. Romanow and S. Floyd. Dynamics of TCP Traffic over ATM Networks. IEEE Journal on Selected Areas in Communications. pp. 633-41, vol. 13, No. 4, May 1995.

[10] H.-Y. Tzeng and K.-Y. Siu. Performance of TCP over UBR in ATM with EPD and Virtual Queuing Techniques. Proceedings of Workshop on Transport Layer Protocols over High Speed Networks, IEEE Globecom, Nov. 1996.

[11] Y. Wu, K.-Y. Siu, and W. Ren. Improved Virtual Queueing and EPD Techniques for TCP over ATM. To appear in IEEE International Conference on Network Protocols, Oct. 1997.

[12] N. Yin and S. Jagannath. End-to-End Traffic Management in IP/ATM Internetworks ATM Forum Contribution 96-1406, October 1996. 